\title{
Suppressed Polariton Scattering in Semiconductor Microcavities
}

\author{
J. J. Baumberg, ${ }^{1}$ A. Armitage, ${ }^{2}$ M. S. Skolnick, ${ }^{2}$ and J. S. Roberts ${ }^{3}$ \\ ${ }^{1}$ Hitachi Cambridge Laboratory, Cavendish Laboratory, Madingley Road, Cambridge CB3 OHE, United Kingdom \\ ${ }^{2}$ Department of Physics, University of Sheffield, Sheffield S3 7RH, United Kingdom \\ ${ }^{3}$ Department of Electronic and Electrical Engineering, University of Sheffield, Sheffield S1 3JD, United Kingdom
}

(Received 26 November 1997)

\begin{abstract}
Ultrafast time-resolved measurements on a semiconductor microcavity distinguish polariton dynamics dependent on the detuning between the constituent photon and exciton modes. Spectral synthesis allows the injection of specific polariton pulses into the sample. Scattering which results in absorption is found to be suppressed in the lower polariton branch by the combined action of normal mode splitting and motional narrowing. The experimental difference between angle and position tuning demonstrates the role of the polariton dispersion in polariton-polariton interactions. [S0031-9007(98)06665-4]
\end{abstract}

PACS numbers: 71.36.+c, 42.50.-p, 42.65. $-\mathrm{k}, 78.47 .+\mathrm{p}$

Manipulating the interaction between light and semiconductors by engineering the electron and photon states has enabled a variety of striking coherent phenomena to be observed in recent years. By embedding a semiconductor quantum well (QW) inside a wavelength scale optical cavity, strong and tunable coupling between the band-gap excitons and photons produces new polariton modes which dominate the optical spectra $[1,2]$. Many experiments have probed these microcavity properties using reflection, transmission, and photoluminescence measurements in both $\mathrm{cw}$ [3-5] and time-resolved [6-8] domains. In the former, the two polariton peaks are spectrally resolved, whereas, in the latter, short pulses impulsively drive both polariton branches producing characteristic Rabi oscillations. These investigations mainly track the escape of polaritons from the microcavity via their leakage through the mirrors.

In this paper we resolve instead the scattering of polaritons into states in which they are irreversibly absorbed and cannot promptly reradiate. Using spectrally tailored pump pulses in time-resolved nonlinear measurements, distinct differences in absorption are uncovered for the two branches of the polariton dispersion. Although the sample reflects equally strongly on either spectral line when the photon and exciton modes are in resonance, polariton scattering in the lower branch is clearly suppressed. Such behavior was predicted for optic phonon scattering of bulk polaritons [9] but inconclusively verified-in contrast, microcavity samples provide direct access to the polariton modes. These data confirm a recent prediction that the lower branch polariton is more effectively decoupled from localized exciton states [10]. Two methods for tuning the polariton resonance by varying the angle of incidence or the cavity frequency are seen to be inequivalent due to the influence of the polariton dispersion.

The semiconductor quantum microcavity (QMC) sample is formed from two distributed Bragg reflectors (DBRs) alternating ten $\lambda / 4$-thick layers of GaAs and AlAs and contains three $100 \AA \mathrm{In}_{0.06} \mathrm{Ga}_{0.94} \mathrm{As}$ QWs, $100 \AA$ apart. The optical cavity length is $\sim \lambda_{\text {ex }}$. Mea- surements on a piece of the wafer near resonance allow both angle and position tuning of the relative energy $(\Delta)$ between cavity and exciton resonances. The simplest model of this system predicts a characteristic anticrossing through $\Delta=0$ of these coupled light and matter oscillators. The mixed normal modes, $|+,-\rangle$, are clearly identified from the energy positions of the dips in the probe reflection spectrum [Fig. 1(a)]. The minimum energy separation of the polariton modes, known as the normal mode splitting, $\omega_{R}=5.7 \mathrm{meV}$, is determined by the coupling strength between the QWs and the optical field in the cavity. In this sample, the short retention time of photons within the QMC leads to a spectral width of the bare cavity mode $(2.2 \mathrm{meV})$ which is more than double the exciton linewidth $(0.9 \mathrm{meV})$. This regime is of interest because significant transmission
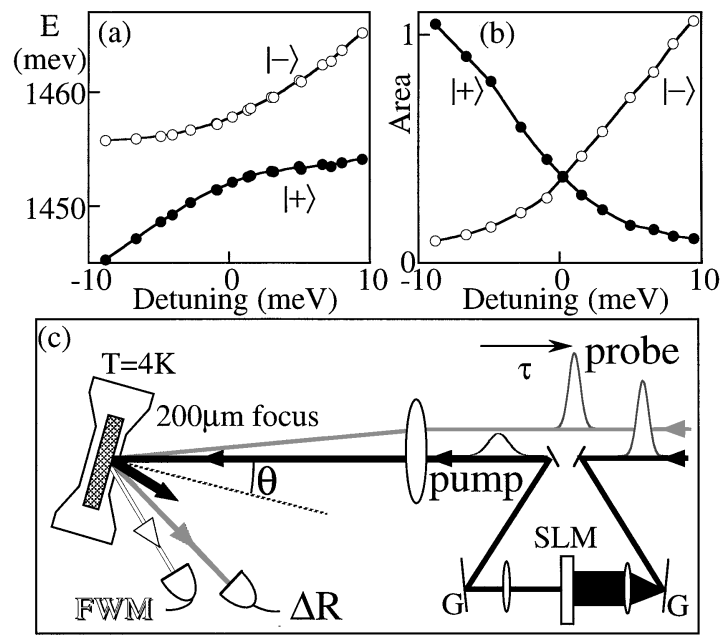

FIG. 1. (a) Spectral positions of the minima in reflectivity as the detuning is swept by translating the sample. Normal incidence $(\theta=0)$ and $T=4 \mathrm{~K}$. (b) Integrated area of the two polariton reflections. (c) Time-resolved experiment: Pump pulses from a Ti:sapphire laser are spectrally filtered using gratings (G) and a spatial light modulator (SLM) and focused onto the sample. Changes in reflectivity and four-wave mixing are recorded simultaneously. 
and absorption occur together (in contrast with higher finesse cavities). On resonance, the two polariton lines have equal integrated strengths [Fig. 1(b)], although the upper polariton linewidth is larger due to the spectrally asymmetric exciton absorption [10].

The absorption technique exploited here uses the sensitivity of the strong QMC reflection to photoinjected carriers which have been irrevocably absorbed. The timeresolved reflection $(\Delta R)$ and two-beam four-wave mixing (FWM) signals induced by a pump pulse are probed simultaneously, with 100 fs pulses from a Ti:sapphire laser [Fig. 1(c)]. Since polaritons in the microcavity escape as photons within several cavity lifetimes, long-lived changes in the reflection spectrum must be due to real carrier absorption. In contrast, identification of absorption from $\mathrm{cw}$ spectral linewidths is complicated by the influence of cavity and disorder contributions [10]. Photocurrent measurements are also nonideal as they require samples with doped DBRs and large electric fields to extract the photoexcited carriers [5]. In order to discriminate the energy dependence of the polariton absorption, the pump pulse is spectrally filtered through a spatial light modulator under computer control, using the method of [11]. Both the amplitude and the phase are controlled within each small wavelength interval (of width $0.35 \mathrm{~nm}$ ) over the $12 \mathrm{~nm}$ pulse bandwidth. Experiments are performed for specific detuning by shaping narrow band Gaussian pulses centered at either the upper or the lower polariton energy. Spectral widths of $2.0 \mathrm{~nm}$ produce longer pulses of $\simeq 700 \mathrm{fs}$ duration. The copolarized pump and probe pulses are focused nearly collinearly (intersecting at $\sim 3^{\circ}$ ensures resonant conditions for both incident and diffracted beams) to a $200 \mu \mathrm{m}$ spot on the sample which is mounted in a continuous flow cryostat at $T=4 \mathrm{~K}$. Data are taken here for both near-normal incidence on the QMC, and for an angle of incidence $\theta \sim 25^{\circ}$. Thus the pump injects polaritons with a tunable in-plane wave vector but which are close in wave vector to those sensed by the probe.

Initially we tune the exciton and cavity modes near resonance $(\Delta=-1 \mathrm{meV})$ and time resolve the reflectivity changes in the probe spectrum, $R_{\text {probe }}$, from an intense ultrashort pump pulse, $P_{\delta}=10 \mathrm{~mW}$. To check that electronic changes last for less than the 12 ns separation of pulses in the mode-locked laser train, and that longlived thermal effects are negligible, we confirm that $R_{\text {probe }}$ is identical when the pump pulse arrives after the probe pulse ( $\tau=-3 \mathrm{ps}$ ) and when the pump is blocked.

Using the full pump bandwidth [Fig. 2(a), $P_{\delta}$ shaded], the incoming short pulse reduces the normal mode splitting and increases the linewidth of the two polariton modes. This implies that the exciton oscillator strength is decreased, as expected from the well-known exciton-exciton interactions produced by photoexcited carriers [12]. Such behavior has been noted before in strongly pumped microcavities [13]. However, the system dynamics here always remains in the low exciton density linear regime and within the strong coupling limit of the QMC. If instead

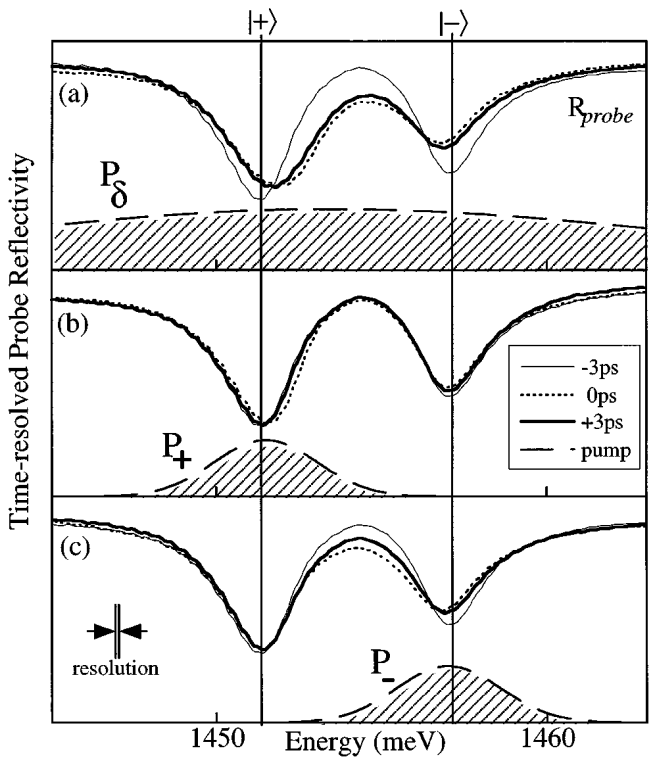

FIG. 2. Transient probe reflection spectra before $(\tau=$ $-3 \mathrm{ps})$, during $(\tau=0 \mathrm{ps})$, and after $(\tau=+3 \mathrm{ps})$ the arrival of the pump pulse (shaded spectrum), $\theta \sim 0$. (a) Unfiltered short pump pulse $P_{\delta}$. (b), (c) Gaussian pulse centered at $|+,-\rangle$.

the pump spectrum is reduced to excite only the lower polariton branch, $|+\rangle$, keeping the same spectral density [Fig. 2(b)], no change is seen in the reflection spectrum once the pump pulse has completely escaped the cavity $(\tau=+3 \mathrm{ps})$. In contrast, pumping the upper polariton line, $|-\rangle$, produces a long-lived reduction in the normal mode splitting and bleaches the polariton line [Fig. 2(c)].

Long-lived effects occur only if the injected polaritons are scattered into optically inactive states, which may be free carriers, excitons at large $\mathbf{k}$, or dark excitonic polaritons. The latter are two modes formed along with the bright polariton mode and also delocalized between the 3 QWs but optically inactive due to their symmetry [2]. However, a much bigger reservoir is provided by excitons with large in-plane momentum that cannot directly radiate $[2,10,14]$ as well as the nonradiative components of localized excitons which do not contribute to the polaritons: the inhomogeneous quantum confinement potential produces a broad energy distribution of localized excitons which each supply some $\mathbf{k}=0$ strength to the polaritons while the residual $\mathbf{k}$ components form localized, optically inactive excitons [15]. Both free high-k excitons and localized excitons can reradiate only by scattering back into polaritons, which is highly suppressed because of their large density of states compared to the low-mass $\left(m^{*} \sim 10^{-5} m_{e}\right)$ delocalized polariton modes. Occupied nonradiative states modify the exciton component of the polaritons through a combination of phase space filling, screening, and exchange interactions. Thus the directly resolved difference between the long-lived nonlinear response of the two spectral lines clearly demonstrates the suppression of absorption due to the lack of polariton scattering from the lower branch. 
Also prominent in Fig. 2(c) is the different response of $|+\rangle$ and $|-\rangle$ to $P_{-}$. Since equal exciton contributions are mixed into the two polariton branches, the nonlinear exciton response should contribute induced changes to both polariton modes. Instead here the upper polariton suffers much stronger nonlinear effects than the lower polariton. To explain this nonlinear reflectivity demands new theoretical models involving a more sophisticated description of the composition of the polaritons to take account of the inhomogeneous distribution of exciton states. The use of spectrally narrow pump pulses with broad band spectral probing is crucial here in identifying the contrasting dynamics of the two polariton modes. This technique demonstrates that microcavity nonlinearities are far from the simple perturbation of the exciton component in each polariton.

To examine the time response further, the spectrally integrated probe reflectivity is measured for the different pump spectra as the time delay is scanned. Both reflection and diffraction dynamics for a range of detunings are shown in Fig. 3 with the pump near-normal incidence. For a short pulse, strong Rabi beating of the polaritons is seen [Fig. 3(a)]. As predicted from integrating the spectral shifts in Fig. 2(a), $P_{\delta}$ reduces $R_{\text {probe }}$. Similarly when selectively pumping $|-\rangle$, a negative $\Delta R$ is also seen [Fig. 3(e)]. However, pumping the lower line [Fig. 3(c)] produces no long-lived absorption until the cavity frequency is above the bare exciton line $(\Delta>0)$. In fact,

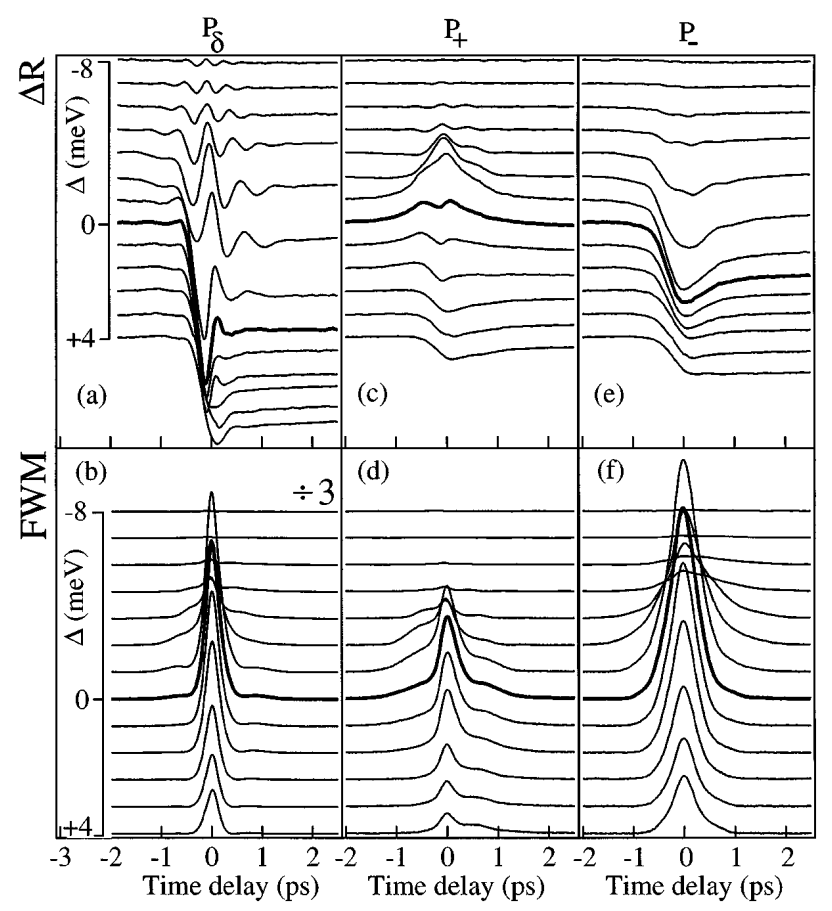

FIG. 3. Time-resolved reflection change and four-wave mixing for pumping: (a),(b) both branches, $P_{\delta}=3 \mathrm{~mW}$; (c),(d) lower branch $P_{+}$; and (e),(f) upper branch $P_{-}$, respectively, vs detuning (increasing downwards, $\Delta=0$ emphasized). The polariton energies are tracked as $\Delta$ is position tuned from -8 to $+4 \mathrm{meV}$. a transient increase in reflectivity is seen for negative detunings, arising from polariton-polariton nonlinearities discussed below. The long-lived absorption signals decay on a $50 \mathrm{ps}$ time scale corresponding to the photoluminescence decay of thermalized polaritons $[3,14,16]$.

The FWM signal arises from coherent nonlinearities which are produced by scattering between the polaritons injected by the pump and probe pulses. It lasts only until the polaritons have leaked out of the DBR mirrors. This diffracted response is also found to be suppressed when pumping the lower branch [Fig. 3(d)] even though the upper and lower polaritons see equally enhanced resonant intracavity fields. This implies that $|+\rangle$ polaritons do not strongly interact with each other. The FWM decay tracks the changes in the polariton escape rate from the cavity, increasing (decreasing) for $|+(-)\rangle$ as the cavity frequency is tuned up to the exciton resonance. This just reflects the relative contribution of the excitons (via dephasing) and photons (via cavity lifetime) to the mixed polariton states. The changes in FWM rise time are, however, not explained by the current model. Coherent diffraction measurements thus further support the qualitatively different nature of the two polariton branches.

To focus on these data more clearly, the total absorption $[\propto \Delta R(\tau=+3 \mathrm{ps})]$ at different detunings is extracted, together with the strength of the nonlinear polariton interactions $\left[\propto \int F W M d t\right]$. These signals are plotted in Fig. 4 for two different incident angles on the sample. In both cases no absorption is seen when pumping the lower exciton branch below the resonant condition [17]. However, at larger incident angles, the FWM signals from the polariton branches become comparable demonstrating that angle tuning and position tuning are not equivalent.

To explain these results, we consider qualitatively the physical processes that can contribute. Three sources of polariton interaction can be identified: $H_{\text {disorder }}$ in which lateral disorder breaks the in-plane translational symmetry and mixes polaritons with localized excitons, $H_{\text {phonon }}$ which scatters the polariton by emission/absorption of acoustic phonons, and $H_{P P}$ due to scattering between two polaritons with $(\mathbf{k} ; \mathbf{k}) \rightarrow(\mathbf{k}+\boldsymbol{\kappa} ; \mathbf{k}-\boldsymbol{\kappa})$. In almost all cases, polaritons will preferentially scatter into localized or high-k exciton states because their density is so much greater than the density of optically coupled polariton states. At resonance or below, $|+\rangle$ absorbs very weakly because it barely overlaps with the density of optically coupled exciton states, $D_{\text {ex }}(\omega)$, which is asymmetrically broadened to higher energy [Fig. 4(e)] by the finite exciton mass in the disorder potential [10]. Hence, the effect of strong coupling between light and matter is to shift the polariton mode below the main band of localized excitons which prevents it from mixing with these optically uncoupled states. In contrast, $|-\rangle$ is resonant with the highenergy tail of excitons leading to fast disorder scattering.

Phonon emission into localized exciton states is also suppressed for $|+\rangle$ since it takes longer than the polariton lifetime, and is inaccessible for $|+\rangle$. At resonance 


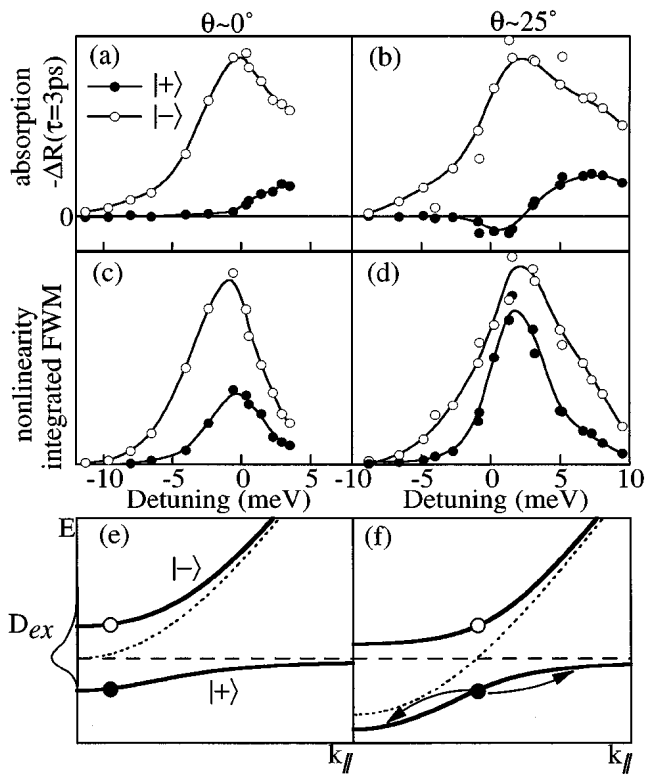

FIG. 4. Extracted long-lived reflection change (a),(b) and integrated FWM (c), (d) for angles of incidence, $\theta=\left(0,25^{\circ}\right)$, respectively, at $T=4 \mathrm{~K}$ using position tuning. (e),(f) Schematic dispersion relations for these $\theta$, with the optical density of exciton states $\left(D_{\mathrm{ex}}\right)$ on the left, the polaritons (solid line), bare exciton (dashed line), and cavity (dotted line) uncoupled dispersions. The points indicate the in-plane $\mathbf{k}$ for resonant excitation in the two geometries.

or below, phonon absorption by $|+\rangle$ into higher energy localized states is restricted due to constraints on energy and momentum conservation, and the small phonon occupation at $T=4 \mathrm{~K}$ [18]. Neither $H_{\text {disorder }}$ nor $H_{\text {phonon }}$ should depend strongly on incident angle: as $\theta$ increases, the relative spectral positions of excitons and resonant polaritons do not change [Fig. 4(f)], so absorption should remain the same, as is indeed observed. Hence, the energy dispersion and consequent decoupling of the polariton and localized states accounts for the lack of absorption in the lower polariton branch, compared to polaritons in the upper branch which have time to scatter before they can escape from the cavity. The differences between the polariton branches also restrict their use for coherent control as recently proposed [19].

The same framework accounts for the FWM signature produced by nonlinear $H_{P P}$ interactions. At normal incidence, two lower branch polaritons cannot scatter off each other because there are no available final states. In contrast, scattering between upper branch polaritons produces significant diffracted signals. At larger angles, phase space becomes more available for both branches [arrows, Fig. 4(f)] tending to equalize the FWM peaks, as observed. This explains why angle tuning and position tuning give different results.

The effects of these nonlinear polariton interactions are visible in the instantaneous spectral changes seen at zero delay in Fig. 2. When the lower line is pumped, it experiences an ultrafast $0.3 \mathrm{meV}$ blueshift at $\tau=0$ without a corresponding shift of the upper line. This also gives rise to the transient increase in $\Delta R$ noted in Fig. 3(c). Similarly when the upper line is pumped, it transiently redshifts by $0.3 \mathrm{meV}$, leaving the lower line almost unmoved. A complete description of this effect requires account of the bipolariton interactions [20]; however, it strongly resembles an ac Stark effect [12]. In a simplified explanation the intracavity field at $\omega_{+}$ both repels and broadens the higher energy bare exciton line, leading to an increase in $\omega_{\text {ex }}$ but a decrease in $\omega_{R}$. Thus $|+\rangle$ transiently Stark shifts to higher energy while $|-\rangle$ does not move. Similarly, pumping $|-\rangle$ repels the exciton to lower energy. Hence, in this regime, enhanced optical nonlinearities can be observed, at the same time as minimized absorption.

In summary, we have been able to demonstrate the suppression of scattering from the lower polariton branch, using ultrafast pump-probe spectroscopy. These experiments show that although the escape of photons from a microcavity can be equal for two polariton modes, their absorption rates can be very different. This suggests the possibility of engineering appropriate cavity lifetimes and localized scattering rates for optimizing nonlinear optical processes in the absence of significant absorption.

We enthusiastically acknowledge Albert Heberle for experimental expertise with the SLM system and David Whittaker for illuminating discussions. This work was partly supported by EPSRC GR/L32187.

[1] C. Weisbuch, M. Nishioka, A. Ishikawa, and Y. Arakawa, Phys. Rev. Lett. 69, 3314 (1992).

[2] V. Savona et al., in New Aspects in Optical Properties of Nanostructures, Phase Transitions (Gordon and Breach, New York, 1998).

[3] R. P. Stanley, R. Houdré, C. Weisbuch, U. Oesterle, and M. Ilegems, Phys. Rev. B 53, 10995 (1996).

[4] P. Kelkar et al., Phys. Rev. B 52, R5491 (1995).

[5] T. A. Fisher et al., Solid State Electron. 40, 493 (1996).

[6] T. B. Norris et al., Phys. Rev. B 50, 14663 (1994).

[7] H. Wang et al., Phys. Rev. B 51, 14713 (1995).

[8] J. Jacobson et al., Phys. Rev. A 51, 2542 (1995).

[9] B. Bendow and J. L. Birman, Phys. Rev. B 1, 1678 (1970).

[10] D. M. Whittaker, Phys. Rev. Lett. 80, 4791 (1998).

[11] A. Weiner et al., Opt. Lett. 15, 326 (1990).

[12] S. Schmitt-Rink, D. S. Chemla, and D. A. B. Miller, Adv. Phys. 38, 89 (1989).

[13] F. Jahnke et al., Phys. Rev. Lett. 77, 5257 (1996).

[14] J.-K. Rhee, D. S. Citrin, T.B. Norris, Y. Arakawa, and M. Nishioka, Solid State Commun. 97, 941 (1996).

[15] R. Houdré, R. P. Stanley, and M. Ilegems, Phys. Rev. A 53, 2711 (1996).

[16] B. Sermage et al., Phys. Rev. B 53, 16516 (1996).

[17] Equal incident powers $P_{+}=P_{-}$were used. Scaling the results with the intracavity power would enhance the discrepancy between $|+\rangle$ and $|-\rangle$.

[18] V. Savona and C. Piermarocchi, Phys. Status Solidi (a) 164, 45 (1997).

[19] D. S. Citrin, Phys. Rev. Lett. 77, 4596 (1996).

[20] M. Kuwata-Gonokami et al., Phys. Rev. Lett. 79, 1341 (1997). 